TM-1470

[SSC-N-373]

\title{
SSC Dipole Vacuum Vessel Support Placement Analysis
}

T.H. Nicol

Fermi National Accelerator Laboratory

P.O. Box 500, Batavia, Illinois 60510

August 1987 
SSC-N-373

SSC Dipole Vacuum Vessel Support Placement Analysis

Thomas H. Nicol

Fermilab

August 1987

\section{INTRODUCTION}

Early SSC dipole model magnets were supported at five points along their length by feet welded to the vacuum vessel. The cold mass was supported at the same five points. The number of supports was determined such that the maximum cold mass deflection between supports was limited to 0.010 inches as specified in the first version of the SSC Design Criteria. The spacing between supports was determined to minimize the sag of the cold mass, given five supports. Refer to figure 1 for an illustration of this configuration. Refer to figures 2 and 3 for illustrations of the resulting cold mass and vacuum vessel deflections. Note that the vertical shift of the plot in figure 2 is due to the compression of the support posts.

For installation and alignment reasons, five external supports overconstrain the installed position of the vacuum vessel. Two external supports suffice to fix the location of a magnet assembly. However, two supports are not sufficient to support the cold mass. The static sag of a cold mass supported only at two points is nearly 0.5 inches. The solution is to retain five internal support posts between the cold mass and vacuum vessel and to eliminate three of the five external feet.

For reasons of economy, it is desireable for the two external feet to be coincident (axially) with two of the internal supports. A stiffening ring is welded to the vacuum vessel at the mounting positions of the internal support posts. These stiffening rings reduce bending stresses in the vacuum vessel when loads are imposed on the cold mass assembly. Stiffening rings are also required at the external foot locations to support the weight of the upper magnet assembly in the over/under installation scheme. If the external supports are not coincident with two of the internal supports, seven stiffening rings are required. If they are coincident, only five are required.

\section{ANALYSIS CASES AND RESULTS}

\section{Trial Cases}

The scope of this work was to determine the positions of the internal and external supports such that the minimum cold mass sag requirements were met, 
only five stiffeners were needed, and that the end deflections of the vacuum vessel did not impair magnet interconnections.

The most recent issue of the Magnet System Requirements document stipulates that the sag of the cold mass due to its own weight may not exceed $0.50 \mathrm{~mm}$ ( 0.020 inches) from its average value.

By leaving the internal support locations unchanged from their original positions $(0.00,+/-136.25$, and $+/-272.50$ inches) and eliminating the external feet at 0.00 and $+/-272.5$ inches, we are able to limit the sag of the cold mass to its original 0.010 inches. To offset the effect of the additional vacuum vessel deflection we need to shim the internal posts at the positions of the eliminated feet. Unfortunately, the resulting sag of the vacuum vessel is such that magnet interconnections are made more difficult. Figures 4 and 5 illustrate this case for the cold mass and vacuum vessel respectively. In figure 4, note that shims have been added to each support post to compensate for the sag of the vacuum vessel and the compression of the posts.

Suppose now that we try spacing the external feet such that we minimize the sag of the vacuum vessel. By moving the external supports to their optimum position, the cold mass sag criterion cannot be met. Figures 6 and 7 illustrate this case. Again shims have been added to each support post to compensate for the sag of the vacuum vessel. The 0.043 inch sag of the vacuum vessel is well within our ability to make interconnections easily, but the maximum deviation of the cold mass deflection from its average value is 0.028 inches $(0.71 \mathrm{~mm})$.

\section{Design B Proposal}

The solution which satisfies all of the applicable contraints lies somewhere between these two cases. Figures 8 and 9 illustrate a case which meets all of the required criteria. The internal supports are located at $0.00,+/-158.00$, and $+/-270.00$ inches. The original $+/-272.50$ inch position was changed to $+/-$ 270.00 inches for magnet interconnection reasons. This new position allows the vacuum bellows to be slid out of the interconnection region during installation. The external feet are located at the $+/-158.00$ inch positions. This is the configuration recommended for Design $B$ magnet assemblies. The average cold mass deflection is -0.010 inches. The maximum deviation from the average is 0.016 inches $(0.41 \mathrm{~mm})$. The sag of the vacuum vessel at the ends of a magnet assembly is 0.196 inches and is considered to be well within the range which facilitates magnet interconnections.

\section{Fallback Option}

There are no serious problems in the two-support scheme proposed above. The non-uniform spacing between internal supports requires that the anchor tie bars be of two different lengths rather than one, but analysis of the anchor system indicates that this has a negligible effect on the anchor's performance. 
The only negative impact is in potential compaction of the 80K MLI at the cryostat ends. The $80 \mathrm{~K}$ shield is supported at five points and has negligible sag. The sag of the vacuum vessel, however, will directly decrease the space allotted to MLI at the top of the shield. This is not currently thought to be a problem. If this position changes, there is a fallback option. By spacing the internal supports at their original positions and placing the external supports at the optimum position for two supports we can effectively eliminate the impact of the vacuum vessel sag. The drawback is that seven stiffeners are required because the two of the internal supports would no longer be coincident with the external feet. Figures 10 and 11 illustrate the cold mass and vacuum vessel deflections resulting from this fallback option.

It should be noted that from a deflection standpoint, this option represents the optimum case for a cryostat with two external supports. The cold mass deflections are unchanged from the Design $A$ configuration and the vacuum vessel deflections are as small as possible, given two supports.

\section{SUMMARY}

Table 1 lists the cold mass and vacuum vessel deflection parameters for each of the cases described above.

Table 1. Deflection Results From Support Analyses.

(all dimensions and deflections are in inches)

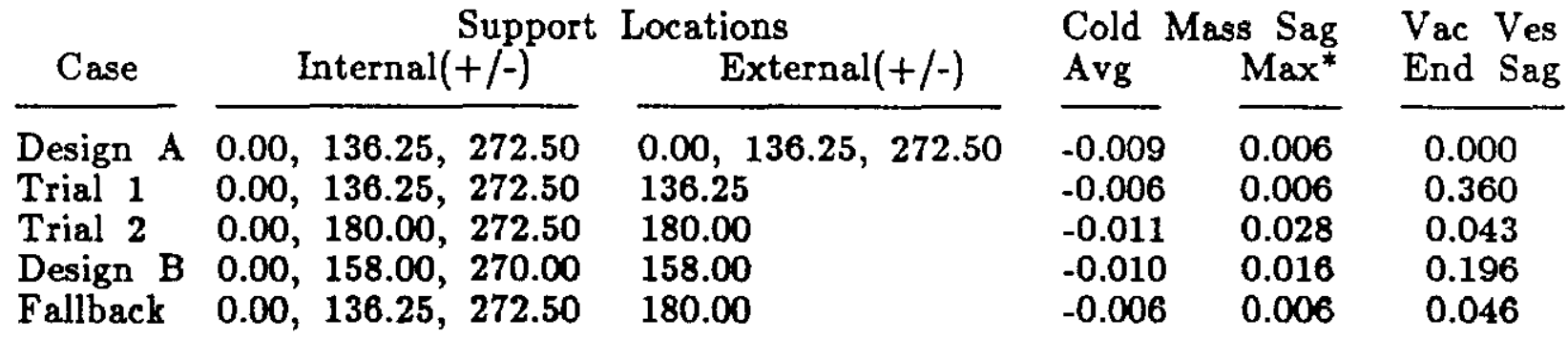

* Max is the maximum deviation from the average cold mass deflection.

Table 2 lists the shims required at the base of each support post for the support configuration proposed for Design $B$.

Table 2. Support post shims required for the Design B proposal.

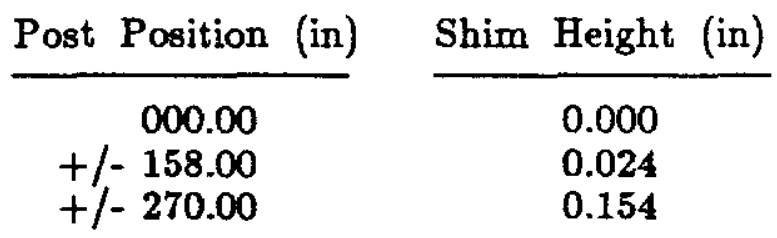


The cryostat configuration represented by this proposal for Design B magnets is illustrated in figure 12 . Aside from the potential compaction of MLI and the required changes in the lengths of anchor tie bars described above, there are no serious flaws in this scheme. There is no reason to delay its implementation.

\section{REFERENCES}

1. "SSC Design 'D' Cryostat Design Criteria, Revision C", July $19,1985$.

2. "Can an SSC Magnet Have Just Two Supports?", D.E. Groom, SSC-N-267, November 25, 1986.

3. "Deflection Analysis for an SSC Dipole Magnet With Two External Supports", T.H. Nicol, SSC-N-283, January 9, 1987.

4. "SSC Collider Magnet System Requirements", Issue No. 2, SSC-MAG-D-101, July 23, 1987. 


\section{Design A Support Placement}

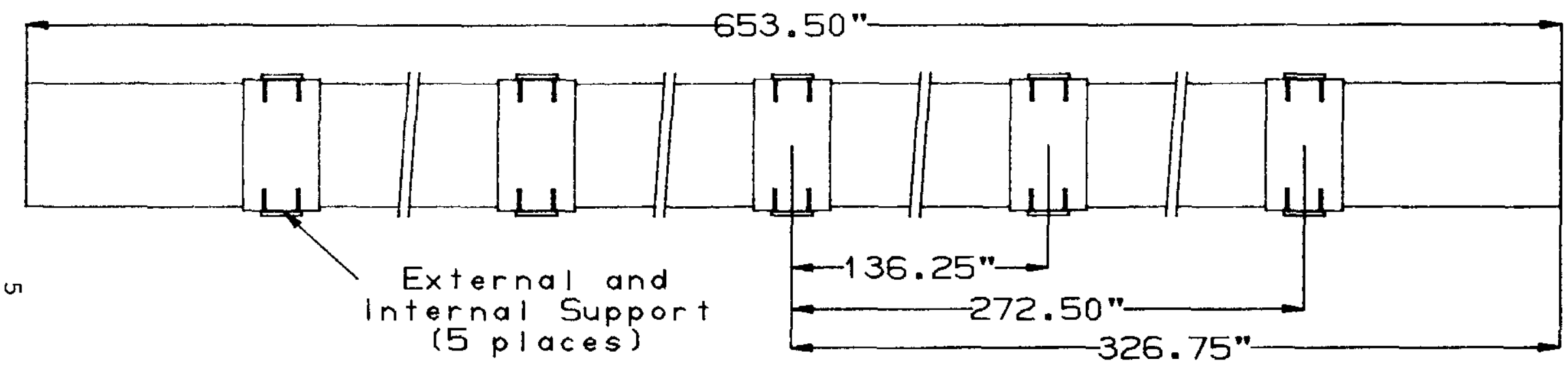

Figure 1 


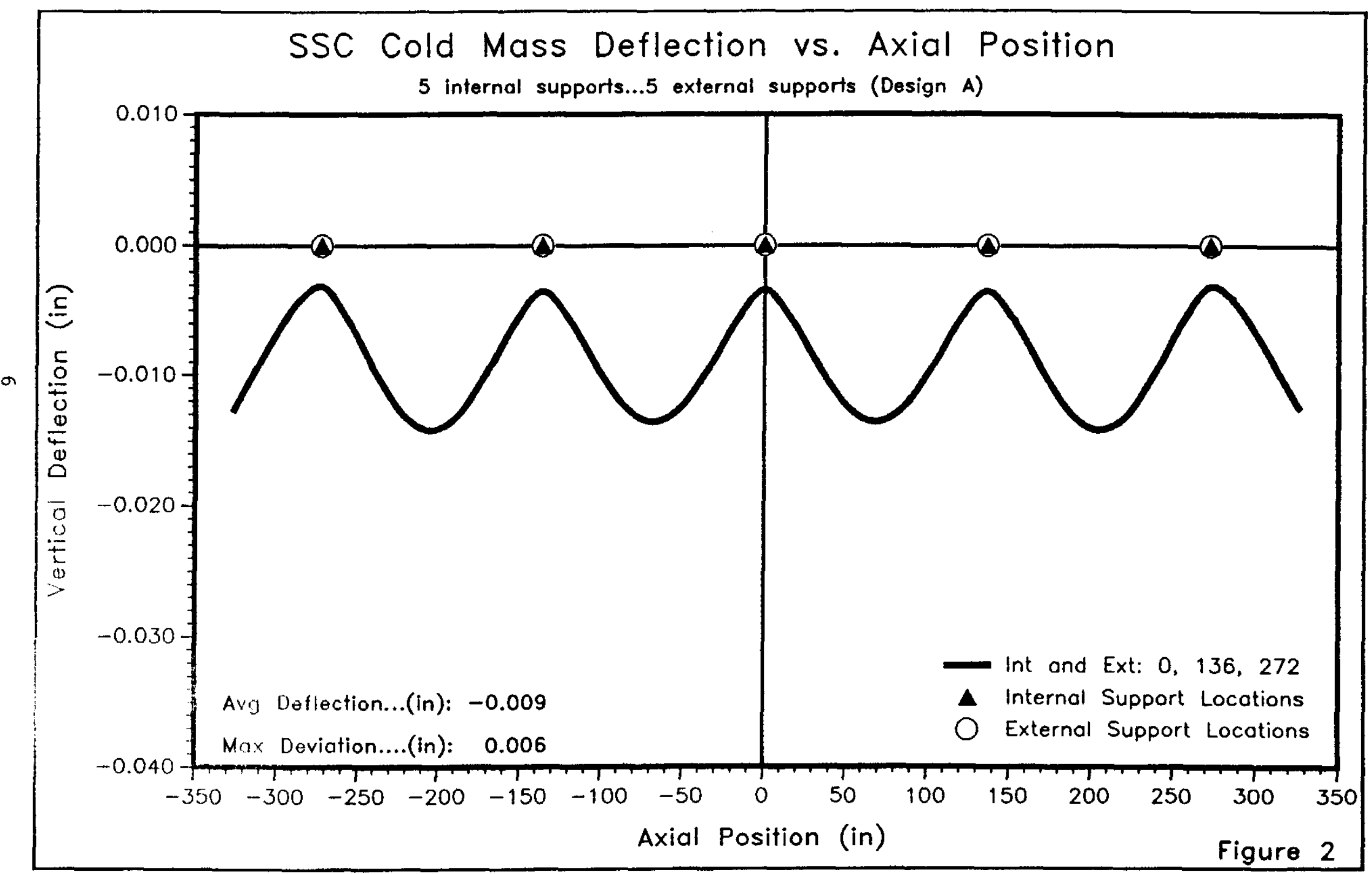




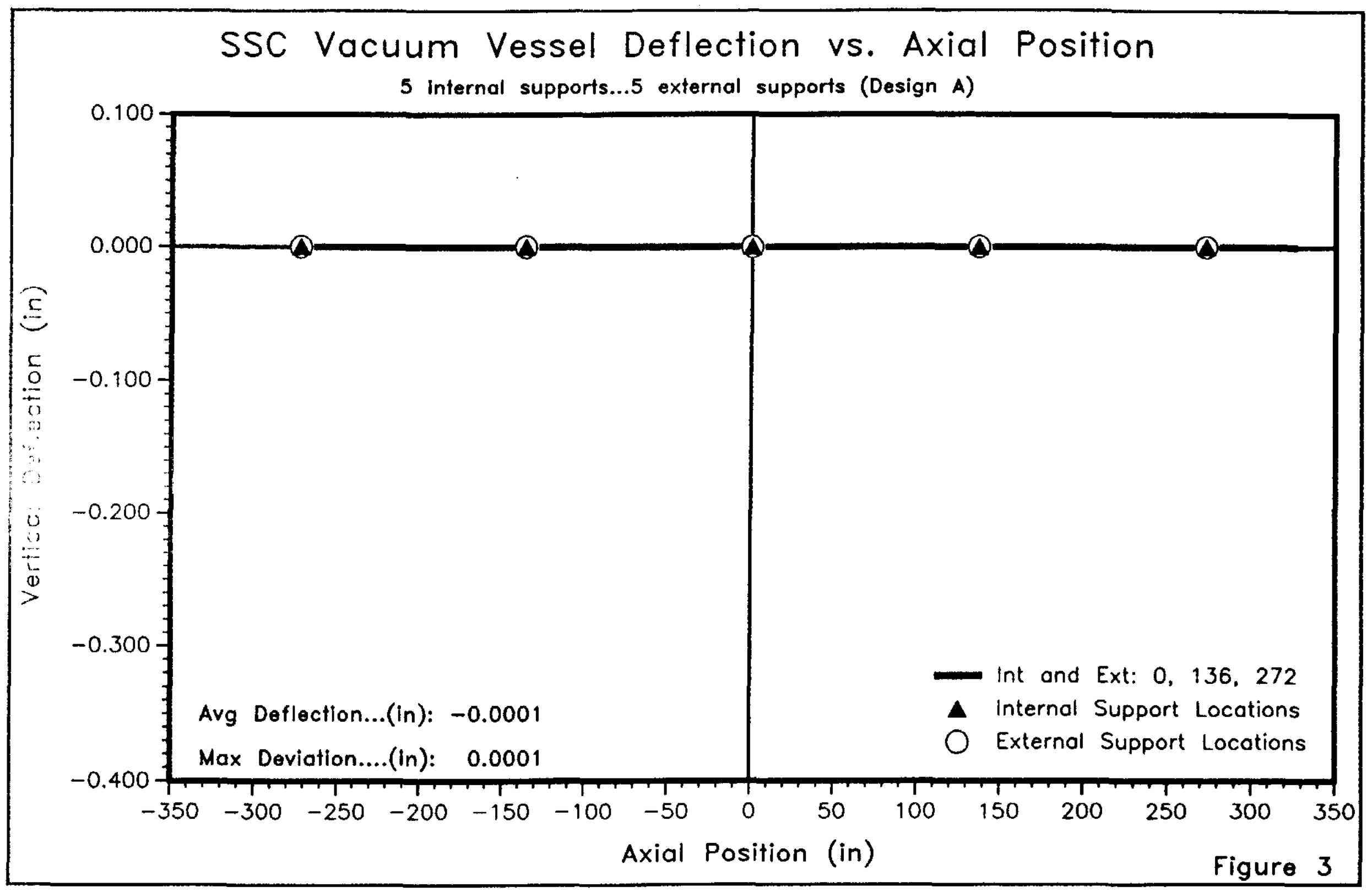




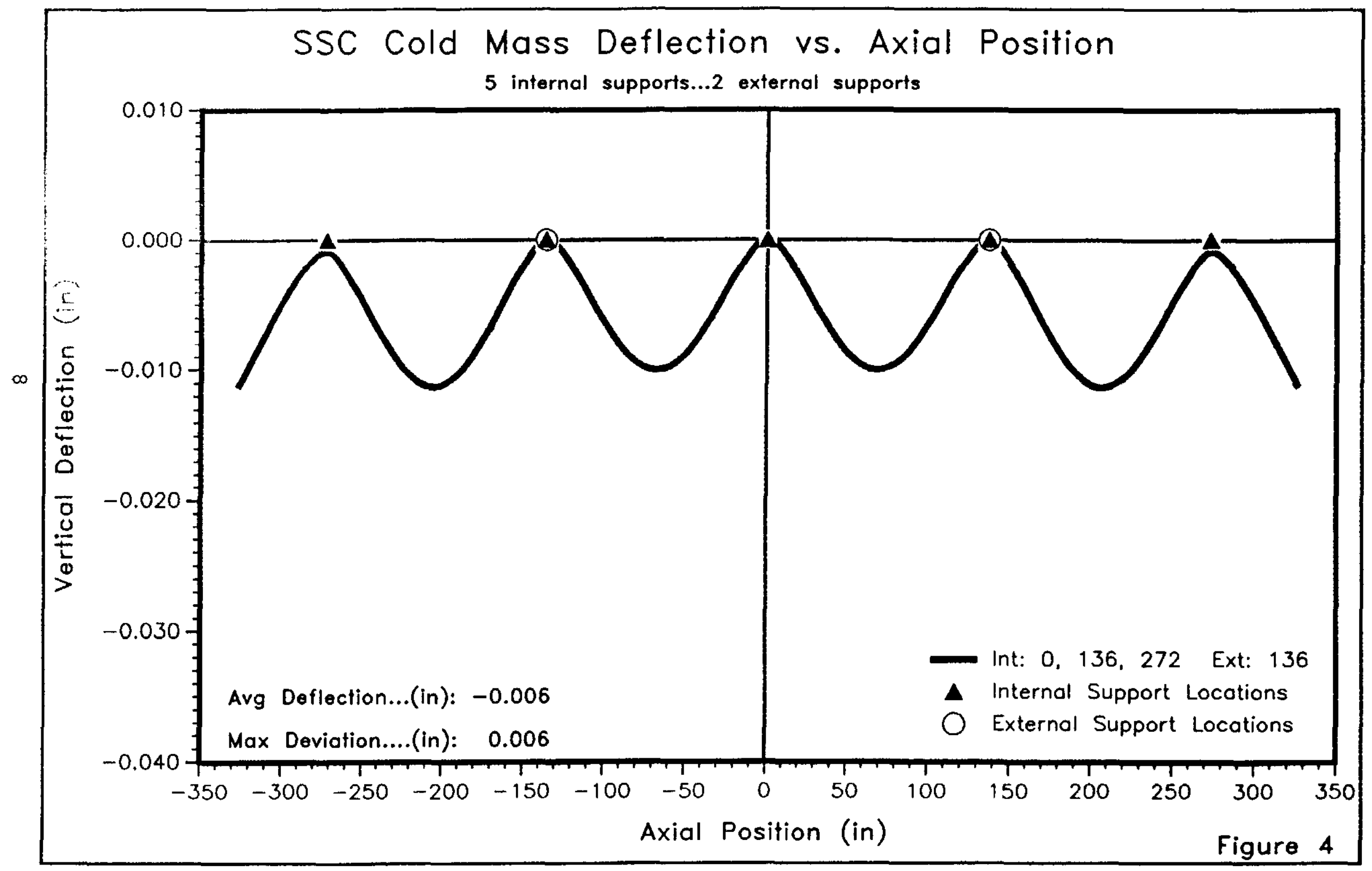




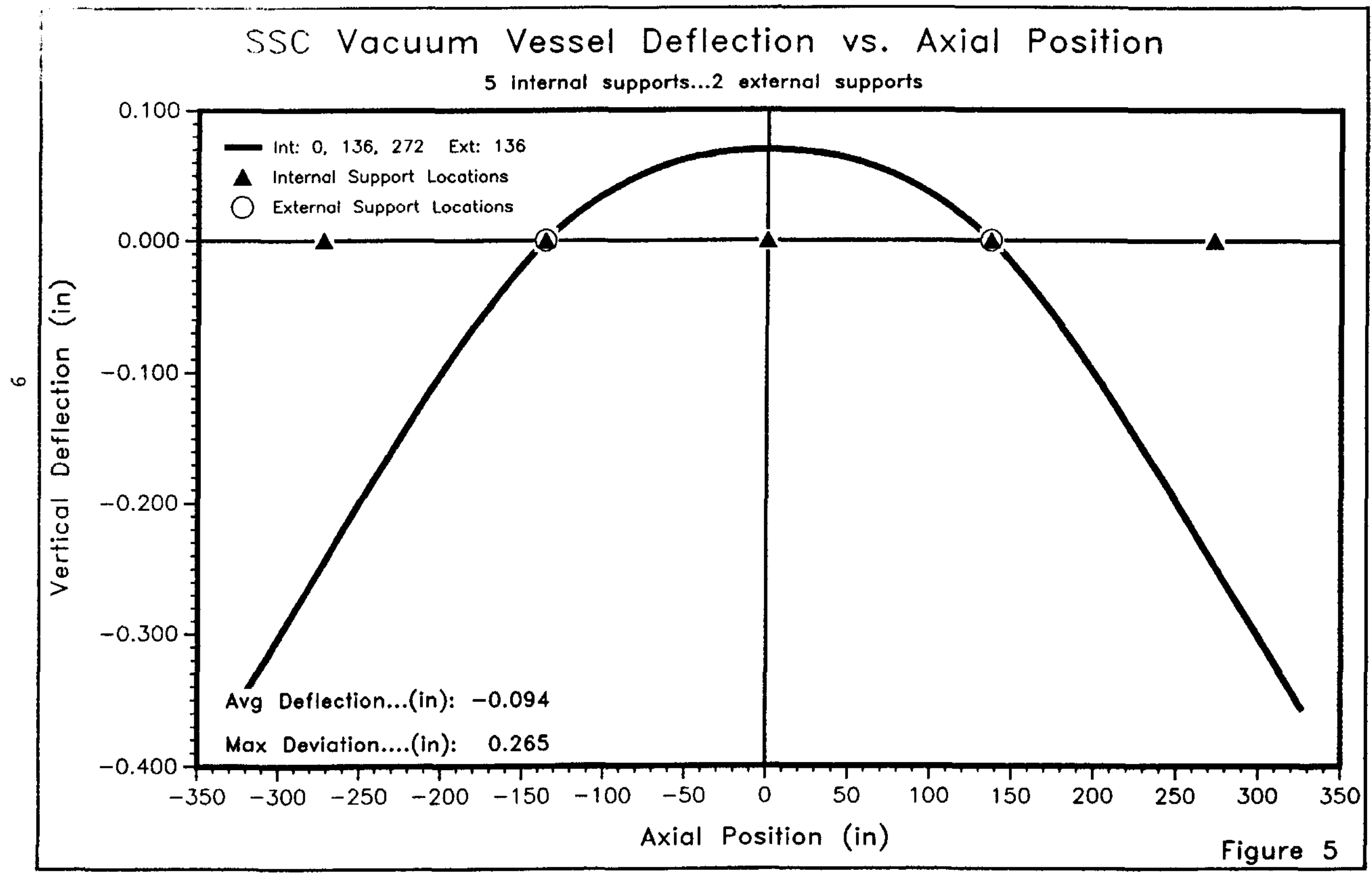




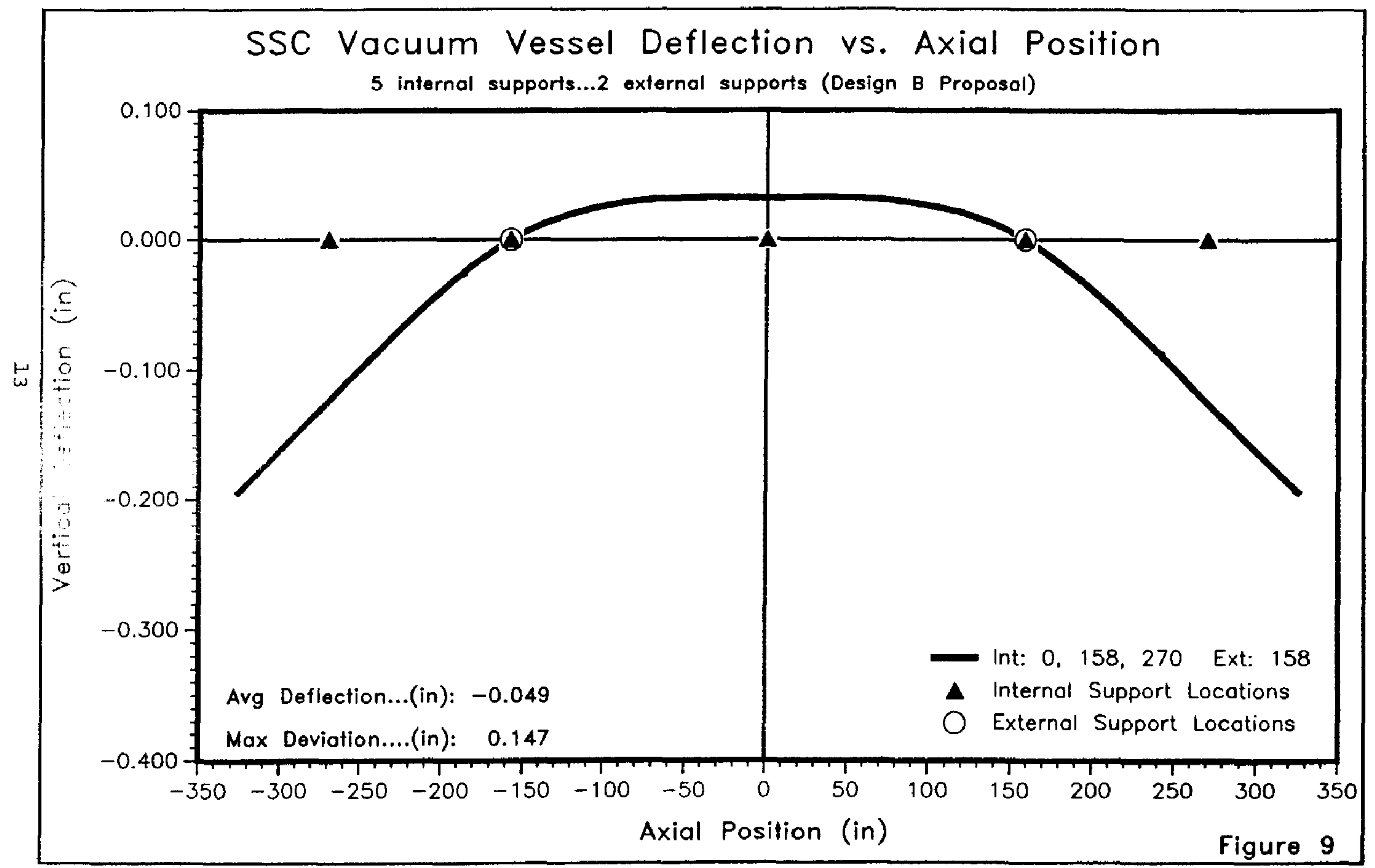




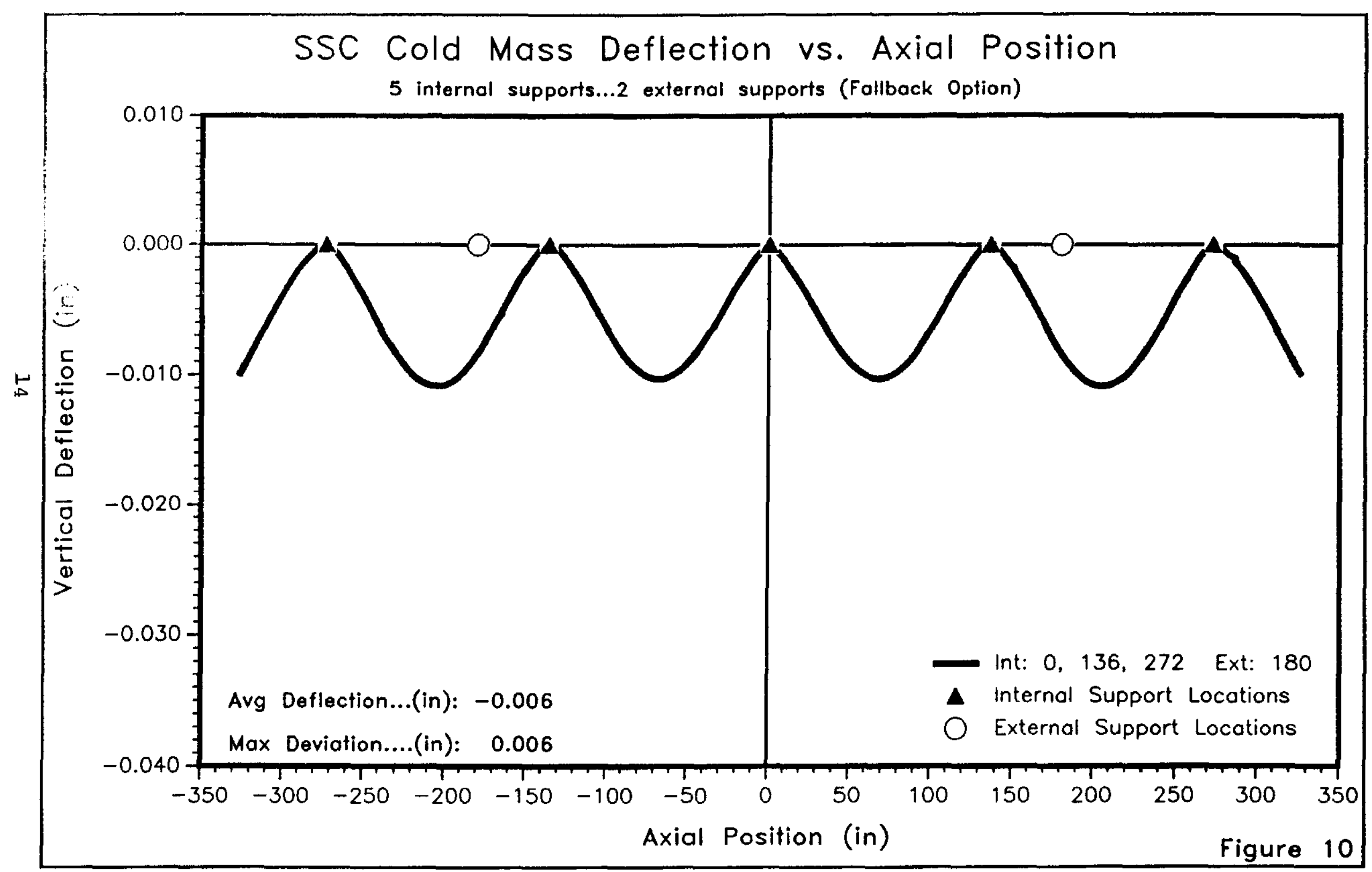




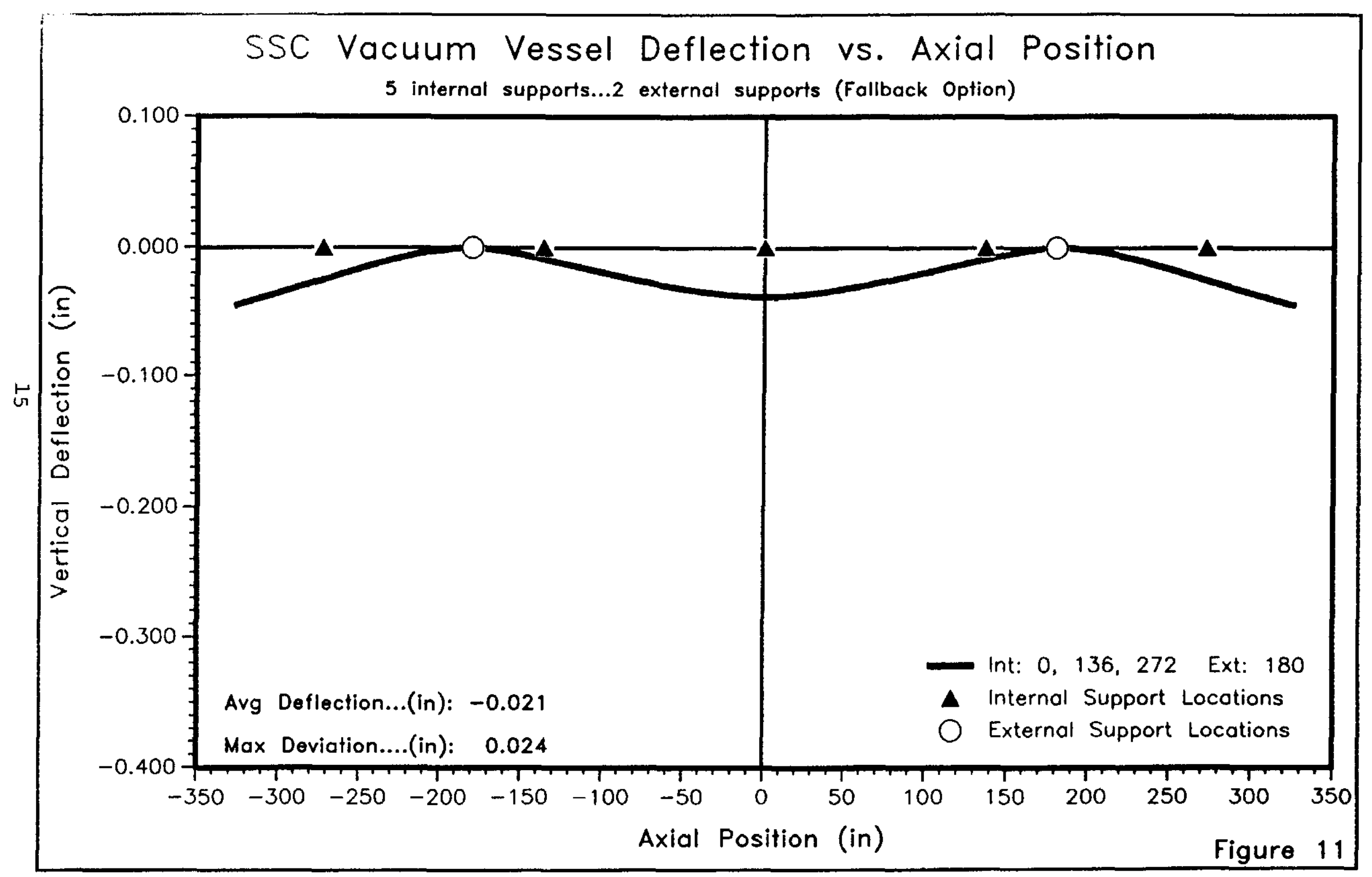


Proposed Design B Support Placement

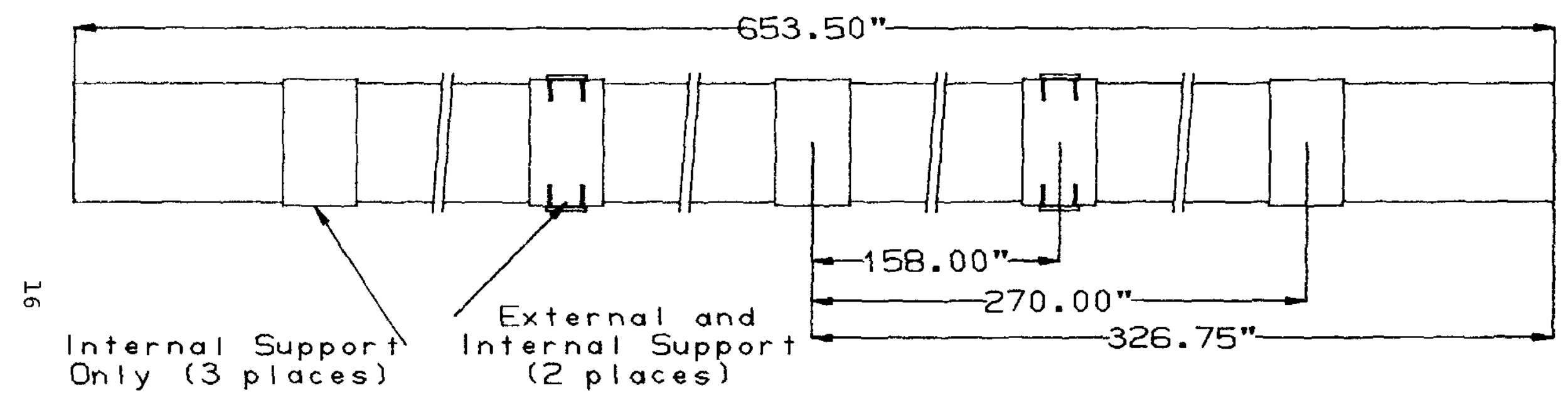

Figure 12 